\title{
Antidepressant Suppression of Non-REM Sleep Spindles and REM Sleep Impairs Hippocampus-Dependent Learning While Augmenting Striatum-Dependent Learning
}

\author{
Alain Watts, ${ }^{1}$ Howard J. Gritton, ${ }^{2}$ Jamie Sweigart, ${ }^{1}$ and Gina R. Poe ${ }^{1,3}$ \\ ${ }^{1}$ Department of Anesthesiology, ${ }^{2}$ Neuroscience Program, and ${ }^{3}$ Department of Molecular and Integrative Physiology, University of Michigan, Ann Arbor, \\ Michigan 48104
}

\begin{abstract}
Rapid eye movement (REM) sleep enhances hippocampus-dependent associative memory, but REM deprivation has little impact on striatum-dependent procedural learning. Antidepressant medications are known to inhibit REM sleep, but it is not well understood if antidepressant treatments impact learning and memory. We explored antidepressant REM suppression effects on learning by training animals daily on a spatial task under familiar and novel conditions, followed by training on a procedural memory task. Daily treatment with the antidepressant and norepinephrine reuptake inhibitor desipramine (DMI) strongly suppressed REM sleep in rats for several hours, as has been described in humans. We also found that DMI treatment reduced the spindle-rich transition-to-REM sleep state (TR), which has not been previously reported. DMI REM suppression gradually weakened performance on a once familiar hippocampusdependent maze (reconsolidation error). DMI also impaired learning of the novel maze (consolidation error). Unexpectedly, learning of novel reward positions and memory of familiar positions were equally and oppositely correlated with amounts of TR sleep. Conversely, DMI treatment enhanced performance on a separate striatum-dependent, procedural T-maze task that was positively correlated with the amounts of slow-wave sleep (SWS). Our results suggest that learning strategy switches in patients taking REM sleep-suppressing antidepressants might serve to offset sleep-dependent hippocampal impairments to partially preserve performance. State-performance correlations support a model wherein reconsolidation of hippocampus-dependent familiar memories occurs during REM sleep, novel information is incorporated and consolidated during TR, and dorsal striatum-dependent procedural learning is augmented during SWS.
\end{abstract}

\section{Introduction}

Memory consolidation is a time-dependent process whereby new, hippocampus-dependent associations become stabilized in the neocortex (McGaugh, 2000). Upon recall, these consolidated memories can become transiently vulnerable to modification before being restored/reconsolidated (Nader and Hardt, 2009). We tested the involvement of rapid eye movement (REM) sleep in the process by which new memories are stabilized (consolidation) and familiar memories are restabilized (reconsolidation) by suppressing REM sleep using a commonly prescribed antidepressant and selective norepinephrine reuptake inhibitor, desipramine (DMI). DMI has a relatively low side effect profile (Goodman et al., 2006) and elevates noradrenaline (NA) at all targets of the locus coeruleus (LC), suppressing REM sleep through inactivation of REM-ON cholinergic pontine neurons (Baumann et al., 1983). Short-term

\footnotetext{
Received Jan. 12, 2012; revised July 9, 2012; accepted July 16, 2012.

Author contributions: H.J.G. and G.R.P. designed research; A.W. and J.S. performed research; A.W., J.S., and G.R.P. analyzed data; H.J.G. and G.R.P. wrote the paper.

This work was supported by National Institutes of Health Grant MH 60670 and the Department of Anesthesiology at the University of Michigan, Ann Arbor, MI. We thank Theresa Bjorness, Victoria Booth, and Jonathon Reasor for their help and advice in the initial stages of the study.

The authors declare no competing financial interests.

Correspondence should be addressed to Dr. Gina R. Poe, 7433 Medical Sciences Building 1, 1150 West Medical Center Drive, Ann Arbor, MI 48109-5615. E-mail: ginapoe@umich.edu.

DOI:10.1523/JNEUROSCI.0170-12.2012

Copyright $\odot 2012$ the authors $\quad 0270-6474 / 12 / 3213411-10 \$ 15.00 / 0$
}

REM sleep deprivation following new hippocampus-dependent learning can impair consolidation, depending on the type of task being performed and the intensity of training (Smith et al., 1998; Bjorness et al., 2005; Walsh et al., 2011), while striatumdependent procedural learning is largely unaffected by REM sleep deprivation (Wimmer et al., 1992; Smith et al., 1998; Bjorness et al., 2005; Hagewoud et al., 2010; Walsh et al., 2011). Individuals using REM sleep-suppressing antidepressants do not self-report marked reductions in memory function beyond those already associated with depression, although some have been documented (Brooks and Hoblyn, 2007). People with depression may rely on alternative learning strategies to offset REM-dependent learning deficits. We chose to address whether REM deprivation affects learning and selection of alternative strategies using DMI as a nonstressful REM sleep deprivation technique that is commonly given to human patients.

Here we report for the first time that REM suppression impairs reconsolidation of a familiar spatial memory, and that the often overlooked transition-to-REM sleep state (TR) is involved in the process of new memory consolidation (Walsh et al., 2011). Both reconsolidation and novel memory consolidation are dependent on normal hippocampal activity (Morris et al., 1986; van der Meulen et al., 2003).

NA acts at $\beta$ receptors to enhance long-term potentiation (LTP) and prevent depotentiation (DP) (Thomas et al., 1996; Katsuki et al., 1997). However, DP is necessary to efficiently in- 
corporate newly learned information into previously established memory networks (Xu et al., 1998; Manahan-Vaughan and Braunewell, 1999; Kemp and Manahan-Vaughan, 2004). Therefore, we posited that forebrain targets of the LC may require LC silence to efficiently remodel hippocampus-dependent memory networks for learning. The LC is normally silent only during REM sleep and at the initialization of sleep spindles (Aston-Jones and Bloom, 1981), which characterize the TR sleep state (Gross et al., 2009). DMI-induced sustained NA elevation in the hippocampus therefore should impair learning-dependent synaptic depotentiation.

As the dorsal striatum is void of NA inputs (Jones and Yang, 1985), we posited that striatum-dependent learning should be immune to noradrenergic manipulations (Nicola and Malenka, 1998) and may benefit from sleep manipulations that impair competing hippocampal strategies (Packard and McGaugh, 1996; Chang and Gold, 2003). Thus, we contrasted hippocampal learning effects of DMI-induced sleep changes with striatal learning effects.

\section{Materials and Methods}

Animals and materials. Thirty-one male Fisher 344 rats aged 6 months and weighing approximately $250-350 \mathrm{~g}$ (Simonsen) were individually housed in plastic cages $(45.7 \times 24.1 \times 20.3 \mathrm{~cm})$ with shaved wood bedding in a climate controlled $\left(22 \pm 3^{\circ} \mathrm{C}\right.$ and $73 \pm 5 \%$ humidity) $12: 12$ $\mathrm{h}$ light/dark cycle chamber. Food and water were initially available $a d$ libitum. Figure $1 a$ shows the detailed order of the experimental procedures. The visible platform version of the Morris water maze task was administered over 2 days to test visual acuity. For this task, animals were placed in a circular tank of $24^{\circ} \mathrm{C}$ water and had to locate and swim to a highly visible platform $2 \mathrm{~cm}$ above the surface of the water to escape. The animals swam 10 trials a day. All but one were selected to continue the study, as their performance was outside 2 SD of the average latency to reach the platform. Proficient performance on this visible platform version of the water maze was essential for their continuation in the study due to the need to use visible distal spatial cues to successfully solve the eight-box maze.

Electrode implantation. The 30 remaining animals were given antibiotic and analgesic and then anesthetized with $60 \mathrm{mg} / \mathrm{kg}$ sodium pentobarbital and placed in a stereotaxic apparatus. Four miniature screw electrodes were bilaterally implanted for electroencephalography (EEG) monitoring: two placed in the frontal cortex and two over the hippocampus. Two electrodes were threaded into the dorsal neck muscles to record electromyography (EMG). All electrodes were crimped to gold pins inserted in a plastic pedestal head stage (Plastics One), and the entire assembly was affixed to the cranium with dental acrylic. Sutures closed the skin around the implant. Animals were monitored until they were responsive and mobile and then returned to their home cage to recover. Antibiotic and analgesic medication was continued for 3 days and then they were video monitored while undisturbed an additional 4 days with ad libitum food and water. All animal procedures were carried out in accordance with the National Institutes of Health Guide for the Care and Use of Laboratory Animals and in accordance with the University of Michigan Committee on the Use and Care of Laboratory Animals.

Motivation. After 7 days of recovery from surgery, food pellets were removed from the home cage and the rats were fed only during pretraining and training sessions to motivate them to run the training mazes for food rewards. The food received during pretraining and testing was a mash of ground rat chow pellets mixed with water. A $2 \mathrm{ml}$ mash supplement mixed with sugar was given per os (p.o.) to all rats after training each day as the vehicle for the DMI (or no-DMI control) and to maintain a minimum of $80 \%$ free-feeding weight. Consumption of DMI-treated or control food was voluntary in most cases and was checked by examining the contents of the supplement bowl after 20 min. Any remaining food was placed at the back of the animal's mouth to insure consumption. Total daily intake of food was equivalent to $\sim 40 \mathrm{cc}$ mash/day to maintain over $80 \%$ free-feeding weight.
Place learning task description. The raised eight-box maze spatial memory task (Poe et al., 2002) is a rectangular track with eight boxes oriented symmetrically around the perimeter of the track. Three of the eight positions contained $0.2 \mathrm{cc}$ of available food in a shallow dish behind a hinged door. All boxes were baited with unreachable food behind a wire mesh just below the door to prevent the successful use of odor-cue location strategies. The familiar maze was configured with the same three baited box positions every day of pretraining and baseline recordings (minimum 11 days) and the 5 days of testing. The novel maze was a reversal task in the same room with the same cue placements. One of the three boxes in the familiar configuration remained baited while the other two formerly baited boxes were changed to other box locations on the maze. To encourage forward motion, the animals were required to make one full clockwise lap before the three baited boxes were refilled. Each training session on each eight-box maze lasted $30 \mathrm{~min}$. Every five laps, rats were removed from the track and placed in a towel-lined pot for 2 min to encourage reliance on hippocampally mediated short-term memory rather than working memory. Errors on each lap were counted when they passed a baited box without stopping (omission error) or inspected or visibly hesitated in front of a box with no accessible food (commission error) and were totaled for each lap (Poe et al., 2002). The experimenter running and scoring the performance of the animal was blind to the drug treatment condition of each animal.

The main outcome measure on the eight-box maze was the number of errors committed on each lap (Fig. 3) and the total errors per lap, per maze, and per training day (Fig. $2 a, d$ ). An average of less than one error per lap over the session was considered as having met performance criterion. Once each animal met performance criterion during the pretraining period, the 5-day testing period began.

Food locating strategies. Without the hippocampus, animals rely more heavily on intramaze cues to successfully perform the task (Roberson et al., 2004; Bjorness et al., 2005). To test the use of nonhippocampally dependent solution strategies, the maze was rotated $180^{\circ}$ after the 10 th lap, the boxes were cleared of any accessible food, and new boxes in the old positions were baited, maintaining the same functional reward positions within the room. Reliance on nonspatial cues to find baited boxes is exhibited by a higher number of errors on lap 11 (after rotation) than on lap 10 (reported in Fig. 3e,f). Working memory was assessed by measuring the decline in errors (or maintenance of low errors) over each five lap set since there were no interruptions in running within each set to disturb the working memory representation (Fig. $3 a-d$ ). Between-session memory consolidation or reconsolidation was assessed by retrieval efficiency between days, which was calculated as the number of errors in the first three laps each day minus the number committed the last three laps of the day before.

T-maze task description. All animals were habituated on a fixed choice T-maze task in a separate room during 3-5 days of the pretraining on the spatial maze task. The day after the first DMI or control treatment, animals were given 15 trials on the T-maze task immediately after training on the eight-box maze for three consecutive days to specifically test procedural striatum-dependent learning abilities during DMI or control treatment conditions. The T-maze used was the fixed choice version, e.g., learning to always turn left at a T-maze junction no matter the starting position (Tolman et al., 1946; Restle, 1957). The maze consisted of four arms joined together to form a "plus" sign. Each arm was 21 " $\times 6$ " $\times 9$ " (length $\times$ width $\times$ height). On each trial, access to one of the arms, chosen semirandomly, was blocked by a wooden barrier and the opposite arm was made the starting position. Thus, from a different one of four possible start positions on each trial, rats were appetitively motivated to advance to the center choice point and either turn right or left to go to the end of the chosen arm for a Cheerio or Froot Loop reward. Rats were rewarded only if they turned in the same direction at the choice point each trial, no matter the location of the start or choice arm relative to the room. The correct turn direction for each rat was determined by their own first choice on the first trial of the first day. Specifically, the placement of the reward was relative to the start position of the rat in an egocentric, not allocentric, reference. For example, if the four arms are placed in the compass positions NSEW and the rat is started in the North arm with the South arm blocked and a right turn is correct for that 


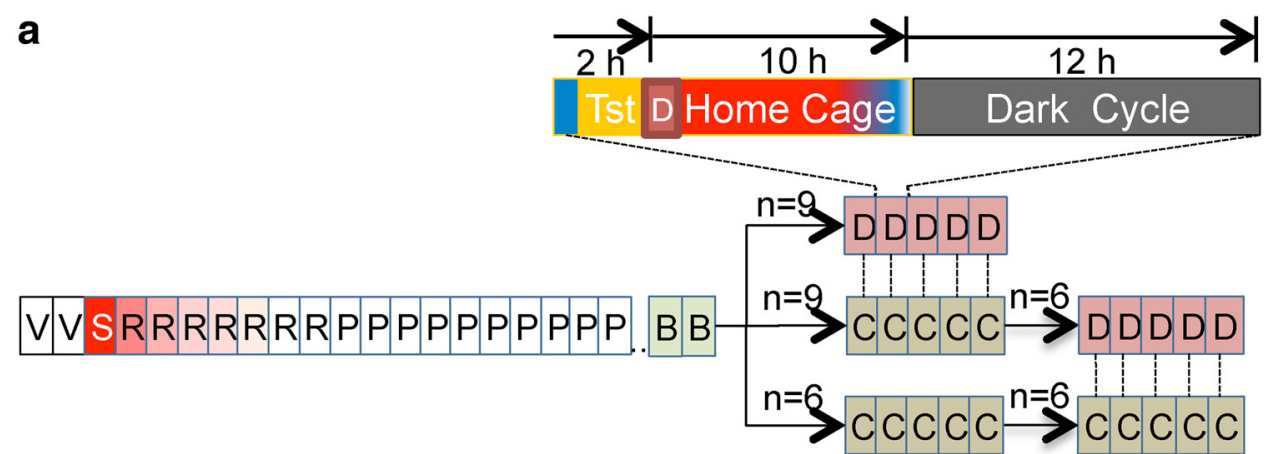

b

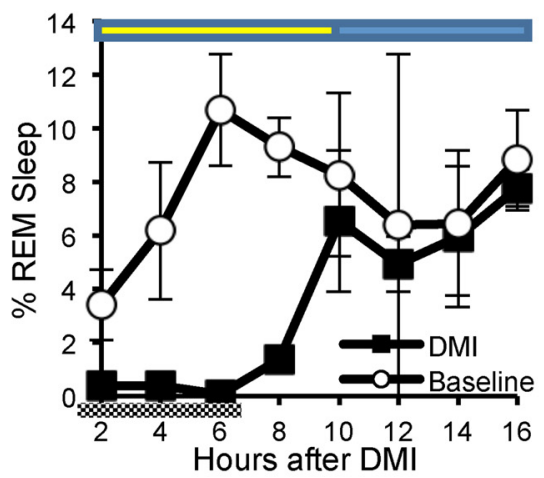

C

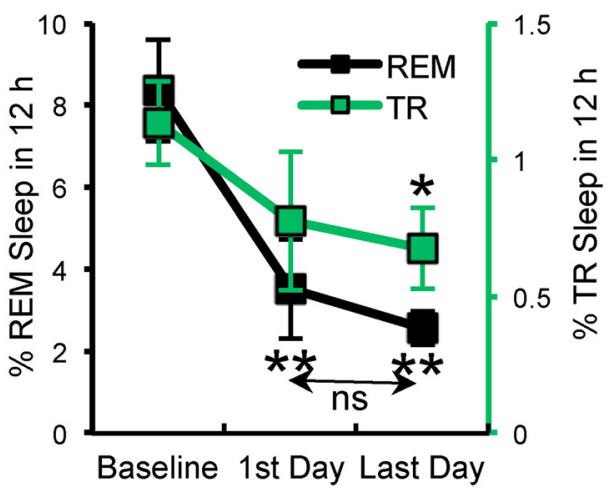

Figure 1. Study design and sleep effects of desipramine. $a$, Study design. Each block represents one day. V, Visual acuity test; $S$, surgery; $R$, recovery; $P$, pretraining (at least 11 days, mean $21.1 \pm 2.4$ days); B, baseline sleep recordings; $C$, control testing $(n=15) ; D$, DMl administration following testing $(n=9$ week $1, n=6$ week 2$)$. One $24 \mathrm{~h}$ experimental day is shown in the dashed outset panel above. The left-most blue box represents the $30 \mathrm{~min}$ after lights-on in the home cage before the animal is removed for training. Tst is the time spent training $(1.5 \mathrm{~h}$ ) on the familiar and novel eight-box mazes and the T-maze. D is the time subjects ingested the $10 \mathrm{mg} / \mathrm{kg}$ desipramine in sweetened mash or control supplement (20 min) before they were returned to their home cages, and their sleep was recorded. The red box fading to blue is the time spent in the home cage during the light cycle $(9 \mathrm{~h} 40 \mathrm{~min}$ ) under the waning influence of DMI. The dark gray box represents the time during the dark cycle when the rat remained in the home cage ( $12 \mathrm{~h}$ ). $\boldsymbol{b}$, Percent time spent in REM sleep following daily testing both before (baseline) and after treatment with desipramine. Data are averaged in $2 \mathrm{~h}$ intervals by condition \pm SEM). Lights-on is depicted by the yellow bar above the graph and lights-off is depicted by the blue bar. The gray bar underscores the complete suppression of REM sleep in the first $6 \mathrm{~h}$ after DMI ingestion. c, Effect of desipramine on REM and TR states during the first and final days of testing relative to the pre-desipramine treatment baseline recording. Percent REM for the $12 \mathrm{~h}$ recording session is shown on the left vertical axis in black. Percent TR for the $12 \mathrm{~h}$ recording session is shown on the right vertical axis in green. ${ }^{*} p<0.01$; ${ }^{* *} p<0.005$, paired $t$ test from baseline. There were no significant differences between the first and final day of treatment in either sleep state.

animal, then the rewarded response is to turn right and end up in the West arm. On the next trial, if he is placed in the South arm he should still turn right at the "T", which would put him in the East arm. Thus, the allocentric map position on which the subject found the reward on any one trial is irrelevant to the task. If the subject uses a hippocampal map to solve the task, he will end up wrong most of the time, as the food moves around from trial to trial relative to the room but is always in the same position relative to the animal. Each trial lasted a maximum of $45 \mathrm{~s}$, with a 60 s intertrial interval spent in a towel-lined clay pot beside the maze. At the completion of 15 trials, the average trial latency and percent correct choices were calculated and compared between Control and DMI treatment conditions. Trial latency and percent correct choices were correlated with sleep parameters of the prior recording day (reported in Fig. 4 and Table 1). Four DMI and three control-treated animals failed to complete all trials and were excluded from the analyses, leaving an $n$ of 11 for DMI and an $n$ of 12 for the control-treated subjects.

DMI dose treatment. An initial DMI dose-response study was conducted to assess the proper dose to suppress REM sleep for $\sim 8 \mathrm{~h}$. Nine rats were given DMI p.o. at either $8(n=3), 10(n=3)$, or $23(n=3)$ $\mathrm{mg} / \mathrm{kg}$ within $20 \mathrm{~min}$ after running the three mazes (familiar eight-box, novel eight-box, and procedural T-maze). At $10 \mathrm{mg} / \mathrm{kg}$, mean REM sleep time suffered a mean $63.9 \%$ reduction (range $22.7 \%$ to $93.8 \%$ ) over the $12 \mathrm{~h}$ light (sleep) period after the maze run compared with each animal's own baseline at the same circadian time. The $10 \mathrm{mg} / \mathrm{kg}$ reduction in REM sleep time was comparable to that of human, whereas the $8 \mathrm{mg} / \mathrm{kg}$ dose had little effect on sleep and the $20 \mathrm{mg} / \mathrm{kg}$ dose produced adverse health consequences (increased heart rate, slowed maze performance). Thus, the $10 \mathrm{mg} / \mathrm{kg}$ dose was used for the remaining experiments. Data from the three pilot rats run at $10 \mathrm{mg} / \mathrm{kg}$ were folded in with the 21 remaining rats tested under control and DMI treatment conditions in pairs run in counterbalanced treatment order on the three mazes (Fig. 1a). Four rats (three paired and one dose-response study rat) were given DMI twice a day, just after running and $12 \mathrm{~h}$ later. Their performance results were compared to those given DMI once per day just after training. No difference was found under the two conditions (repeated-measures ANOVA, $p=0.505$ ), so data were collapsed across methods for statistical analyses and all further experiments were conducted with once per day administration after training. Nine of the $10 \mathrm{mg} / \mathrm{kg}$ DMI-treated rats were recorded in their home cages during all nontraining times and their records analyzed in detail for baseline and DMI sleep characteristics (Fig. 1b-d) and task performance relationships (Figs. $2 b, c, e, 4,5 d$ ).

Sleep recording/scoring/analysis. A shielded cable (Plastics One) from the headstage was connected to a commutator within the home cage to allow free range of motion while recording EEG and EMG signals. The commutator was connected via a shielded cable to a Lynx 8 patch panel and amplifier units (Neuralynx) and to the AD data acquisition system (M. Wilson, Massachusetts Institute of Technology, Cambridge, MA). After three days of habituation to the recording conditions, training began on the eight-box and T-mazes at circadian time $0.5 \mathrm{~h}$ after lights on for $1 \mathrm{~h}$ each day. EEG and EMG recordings began within $1.5 \mathrm{~h}$ of the training session and continued during all non-running times on each of the last 2 days of the pretraining period (baseline) and days $1-5$ of testing in nine rats for comparisons of sleep and performance parameters. 
Sleep/waking states were first scored automatically from EEG and EMG recordings. Every $10 \mathrm{~s}$ epoch was assigned a state of active waking, quiet waking, slow-wave sleep (SWS), transition to REM, or REM sleep by an inhouse automatic sleep scoring program (Gross et al., 2009), and then the scored states were examined and confirmed or corrected after visual inspection by an expert human scorer blind to the experimental condition of the animal. SWS could not be clearly distinguished in one subject while another showed outlier $(>2$ $\mathrm{SD}$ above the mean) quantities of REM sleep during the baseline period and were excluded from the relevant analyses, yielding an $n$ of 8 for most comparisons and an $n$ of 7 for the SWS versus REM comparison (Fig. 4h).

Statistics. Parametric tests such as $t$ tests and ANOVAs were run after tests for normality of the data showed no violations. The nonparametric Wilcoxon sign-rank test was run on the comparison of the number of laps run for DMI and control rats after the DMI lap distribution failed the normality test (median $=15$, range $=11-27$ laps, skewed high, $p=0.0003$ ). Paired $t$ tests were used to evaluate significance ( $n=8, \alpha=0.05$ ) of sleep state changes from baseline to DMI conditions in the same animals (Fig. $1 b-d$ ) and to compare lap 10 versus lap 11 errors within each group $(\alpha=0.05)$ (Fig. $3 e, f)$. A $t$ test was used to compare body weights between DMI and control groups on the last of the 5 day test period. Repeated measures multivariate analyses of variance (MANOVAs) $(\alpha=0.05)$ were performed on the number of errors/lap over testing days $1-5$ and between groups (Fig. 2a,d). Familiar DMI week data were not analyzed from one rat because one of the familiar baited box positions had been inadvertently changed during the prior week, putting the familiarity of the familiar maze during DMI test week into question. Thus, the familiar DMI group statistic was run with an $n$ of 14 rather than 15. Familiar maze data from the first day of 2 DMI/Control pairs was discarded because their familiar day 1 errors exceeded criterion (i.e., they made more than one error per lap), moving the $n$ for the first familiar day analyses in both groups to 13 from 15. The performance metrics that were correlated to percent sleep state parameters during baseline and testing days were the percent of correct choices and latency on the T-maze task over each day for each rat as well as improvements across the testing days for the familiar and reversal mazes $(n=8, \alpha=0.01$ to correct for multiple comparisons). A repeated measures MANOVA test was run on T-maze improvement with DMI treatment on the 3 days following the first DMI treatment. Normality tests were run using JMP software (SAS). Paired $t$ tests were done using Microsoft Excel 2004 Data Analysis Add-In pack (Microsoft). MANOVAs were run with SPSS (IBM), and Pearson's correlation matrix statistics were run using StatPlus (AnalystSoft).

\section{Results}

\section{DMI inhibits REM and TR sleep}

DMI taken orally reduces REM sleep for $5-8 \mathrm{~h}$ in patients being treated for depression (Shipley et al., 1985). A $10 \mathrm{mg} / \mathrm{kg}$ DMI dose p.o. ingested by our subjects suppressed REM sleep for $\sim 8 \mathrm{~h}$ (Fig. $1 b, p=0.0006$ repeated measures MANOVA) with no discernable impact on health or ambulatory activity. Continuous recordings in eight subjects showed that time spent in REM sleep went from an average of $8.35 \pm 1.24 \%$ during the $12 \mathrm{~h}$ light phase (measured on the second day of baseline conditions) to $3.51 \pm$
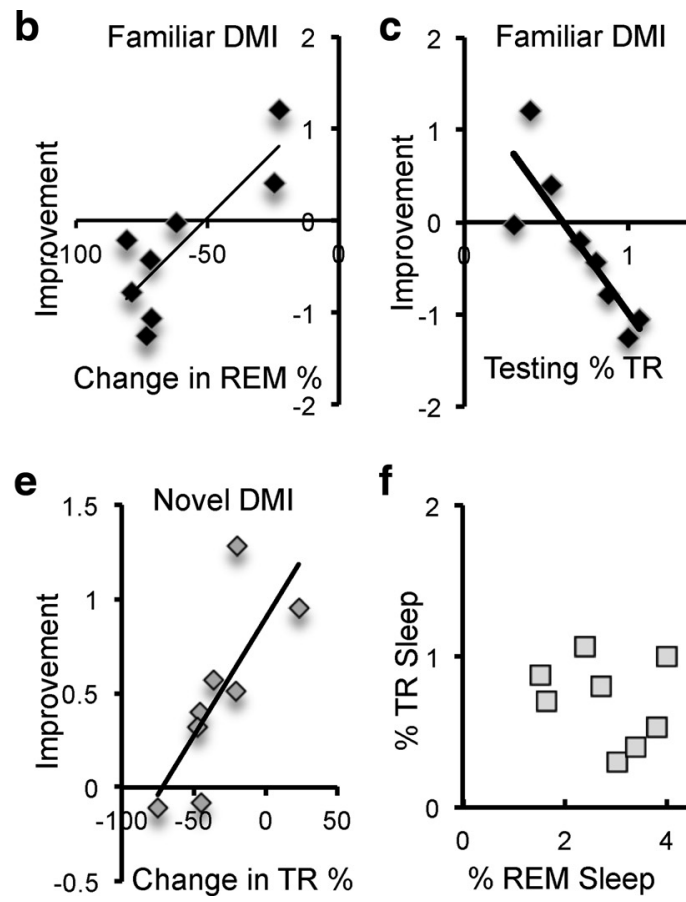

Figure 2. Desipramine effects on consolidation and reconsolidation. $\boldsymbol{a}$, Effect of $10 \mathrm{mg} / \mathrm{kg}$ desipramine (filled squares) on errors per lap in a familiar spatial maze over 5 continuous days of testing relative to control-treated animals (CON; open circles). $\boldsymbol{b}$

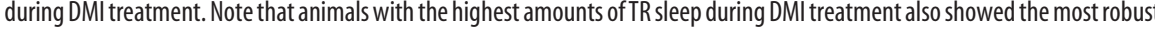
me to control treatment, and DMl-treated animals did not reach criterion of 1 error/lap during the 5 days of testing. Relationship between TR state changes and novel maze performance. Improvement in errors/lap is shown on the vertical axis and percent change in TR state quantities during DMI treatment relative to baseline recordings are shown on the horizontal axis. $\boldsymbol{f}$, Nonsignificant correlation between REM and TR sleep states during desipramine testing.

$1.22 \%$ after administration of DMI $(p=0.00038$, repeatedmeasures ANOVA, baseline vs DMI treatment day effect; Fig. $1 b$ ). Three of every four rats reduced their REM by $72.7 \pm 2.7 \%$ in the $12 \mathrm{~h}$ light phase, while the other quarter reduced REM sleep by $23.6 \pm 0.9 \%$. Within the first $6 \mathrm{~h}$ post-training, i.e., the REM sleep critical window found for this training regimen in a previous study (Bjorness et al., 2005), REM sleep was reduced $95.6 \pm$ $0.22 \%\left(p=3.84 \times 10^{-8}\right)$ consistently across testing days $(p=$ 0.95 , paired $t$ test on first vs last day; Fig. $1 c$ ), suggesting no REM sleep adaptation to desipramine was present during the critical window from the first to the last day of testing. DMI administration also produced a significant decrease ( $p=0.03$, paired two-tailed $t$ test) in the number of REM episodes relative to the same circadian time under baseline sleep conditions (data not shown). Unexpectedly, we found that DMI also attenuated the amount of TR sleep by a range of $13-75 \%$ in the first $12 \mathrm{~h}$ after treatment, with the exception of one animal that showed a 23\% increase in TR under DMI. Mean losses in the TR state under DMI were $30.8 \%$ on average (paired $t$ test, $p=$ 0.016 ; Fig. 1c). There was no correlation between the DMI suppression of TR and REM sleep (Fig. 2f).

\section{DMI treatment does not impair activity or motivation}

Both DMI and control groups ran a similar number of laps on the eight-box mazes each test day and between days (novel maze: control 14.8 laps vs DMI 14.2 laps, Wilcoxon sign-rank test, $p=$ 
0.9387, first day 14.8 laps vs last day 14.3 laps, $p=0.365$; familiar maze: control 18.8 laps vs DMI 18 laps, $p=0.330$, first day 18.3 laps vs last day 18.5 laps, $p=0.461)$. Controls and DMI-treated rats also did not significantly differ in body weight (day 5: control, $336 \pm 14.8$ mean grams \pm SEM; DMI, $326.8 \pm 9.23 ; p=0.389, t$ test), indicating that total maze sampling experience and task motivation did not differ between groups.

\section{DMI impairs novel spatial learning and familiar reconsolidation}

Under DMI treatment, subjects showed significant performance deficits on familiar maze reconsolidation and novel maze consolidation. A general linear model with repeated measures showed a performance deficit with DMI treatment (Fig. $2 a, d ; p=0.001$ ). Although performance was overall better on the familiar versus novel maze (Fig. 2, $a$ vs $d ; p=0.000$ ), DMI had the same negative effect on spatial memory performance in both mazes (no drug by maze interaction, $p=0.759)$. Within subjects there was a powerful learning effect over training day $(p=0.000)$ that was dependent on whether the maze was novel (improved daily performance; Fig. $2 d$ ) or familiar (insignificant daily improvement or significant decline; Fig. $2 a$ ), as revealed in the day by maze interaction $(p=0.000)$. This day effect persisted independent of DMI administration (no day by drug interaction, $p=$ 0.143 , and no day by drug by maze interaction, $p=0.705$ ).

\section{Reconsolidation impaired by DMI treatment}

Performance measured by errors per lap significantly worsened on the familiar maze each day when training was followed by 10 $\mathrm{mg} / \mathrm{kg}$ desipramine (Fig. $2 a$, first-order polynomial of DMI line, $\left.R^{2}=0.75\right)$. By contrast, performance under control conditions held steady (repeated measures MANOVA, DMI vs control, $p=$ $0.005)$. There was no performance difference between groups on the first day of the familiar maze before DMI was administered ( $p=0.34, t$ test), but by day 5 the performance difference between groups was a full error per lap ( $p=0.001, t$ test). Familiar maze performance was correlated with the amount of REM sleep preserved under DMI treatment (Table 1.9). The subjects for whom DMI attenuated REM sleep by only one quarter showed some improvement (Fig. 2b), while the remaining majority of subjects whose REM sleep was attenuated by about $75 \% \pm 3 \%$ in the $12 \mathrm{~h}$ after DMI treatment grew steadily worse each day. The profound but limited range of REM sleep attenuation likely restricted the range for REM sleep correlation calculations. However, the strongest correlation between sleep measures and performance on the familiar maze was a negative one (Table 1.8); those subjects under DMI treatment whose familiar maze performance worsened the most over the 5 days were those that spent the most time in TR (Fig. 2c), and those subjects with the best performance retention had the least amount of time spent in TR. Thus, the more TR was preserved during treatment, the worse the reconsolidation of a familiar memory.

\section{Novel learning impaired by DMI treatment TR loss}

For novel spatial learning on the reversal task, the DMI group never reached criterion, always performing $>1$ mean error/lap even after 5 days (Figs. $2 d, 3 c$ ). Unexpectedly, performance deficits under DMI treatment were not correlated with the rather uniform level of REM sleep suppression during new learning (Table 1.10) but instead were highly correlated with drops in TR under DMI treatment (Fig. 2e, Table 1.7). Thus, unlike familiar memory reconsolidation, the highest performance gains on the novel maze during DMI treatment were made by those animals

\section{Table 1. Correlation coefficient matrix}

\begin{tabular}{lrl}
\hline Variable versus variable & \multicolumn{1}{l}{$R$} & \multicolumn{1}{l}{$p$} \\
\hline 1. Percent correct versus latency & -0.953 & 0.0003 \\
2. REM baseline versus percent correct & 0.923 & 0.0011 \\
3. QS (DMI) versus percent correct & 0.910 & 0.0017 \\
4. \% change in QS versus latency & -0.895 & 0.0027 \\
5. QS (DMI) versus latency & -0.879 & 0.0041 \\
6. REM baseline versus latency & -0.872 & 0.0047 \\
7. Percent change in TR versus novel improvement & 0.867 & 0.0053 \\
8. TR (DMI) versus familiar improvement & -0.853 & 0.0073 \\
9. Percent change in REM versus familiar improvement & 0.833 & 0.0103 \\
10. Percent change in REM versus novel improvement & 0.362 & 0.3721 \\
\hline
\end{tabular}

1. Correlations between latency and percent correct measures on the T-maze. All significant sleep versus performance comparisons described in the text are shown for the T-maze (lines 2-6) and spatial mazes (lines 7-9). To correct for multiple comparisons an $\alpha=0.01$ was selected. No waking parameters were significantly correlated with T-maze or eight-box maze performance metrics, although there was a positive trend between quiet waking and procedural memory (Figure $2 d$ ). 2. The amount of REM sleep during the baseline recording was most correlated to the percent of correct choices and speed of trial completion on the T-maze. 3. The amount of quiet sleep (QS) during desipramine administration was correlated with both the percent correct choice and speed of completing the T-maze trial. 4. The larger the increase in QS after desipramine, the faster the T-maze trial completions. 5. The more QS obtained under DMI, the faster the trial completions. 6. The more REM sleep at baseline, the faster the trial completions. 7. On the eight-box maze the degree of improvement in the novel maze under desipramine treatment was most correlated with the preservation of the transition to REM sleep state as shown in Figure 2e. 8. Those animals whose performance on the familiar maze was best preserved under desipramine treatment were those with the smallest amount of TR sleep, as shown in Figure 2c. 9. Those animals with the largest desipramine suppression of REM sleep had the largest losses during familiar maze reconsolidation (Figure 2b). 10. Improvement on the Novel maze was not significantly correlated with any other sleep parameter, e.g. REM sleep, as listed.

with the smallest declines in the transition to REM sleep (TR) state. Antidepressant action to suppress REM sleep is well known, but its effect on "ascending" stage 2 spindle-rich sleep, called the transition to REM (TR) in the rat, is undocumented. The subjects with the least percent reduction in TR following DMI treatment showed novel learning performances most similar to the control condition, improving performance by an error or more per lap across the 5-day period on the novel maze (Fig. $2 d$ ). Subjects with declines in TR under DMI treatment showed substantially slower learning rates in this novel learning condition $(\sim 0.5$ errors/lap or less improvement; Fig. 2e).

\section{Spatial memory consolidation/reconsolidation impaired, not working, short term, or procedural memory}

Significant improvements (average of $\sim 1$ error/lap) were seen between the first and final lap within any single training session for both control and DMI treatment conditions. On the familiar maze, on average, DMI-treated subjects improved $1.13 \pm 0.20$ errors across the 15 laps (Fig. $3 a, c$ ), and controls improved $0.96 \pm 0.23$ (mean \pm SEM) within the 15 lap session (Fig. $3 b, d$ ). There was no day effect (ANOVA $p=0.7132$ ) or group effect (ANOVA $p=$ 0.5762 ) for this within-session improvement. On the novel maze, both control and DMI treatment conditions also improved performance within session (control $0.92 \pm 0.36$, DMI $0.71 \pm 0.30$ ) with no group effect (ANOVA, $p=0.6423$ ), no day effect ( $p=0.2031$ ), and no day by group interaction $(p=0.996)$. These within-session improvements in the familiar and novel task revealed an intact working memory (unbroken laps 1-5, 6-10, and 11-15) and a functional short term memory (spanning the 2 minute breaks every 5 laps) despite the prior day's DMI treatment or sleep effects thereof.

Instead, the source of the learning deficit for DMI-treated animals on both the familiar and novel mazes was poor overnight retention. A higher number of errors per day in the first few laps revealed deficits in reference memory between days. DMI-treated subjects performing on the familiar maze were unable to effectively reconsolidate the familiar context as evidenced by an average of $1.19 \pm 0.29$ more errors in the first lap compared with the last lap of the prior day (MANOVA drug effect $p=0.0001$ ). An 
increase in the number of errors on the first few familiar maze laps can be seen from day 1 to day 5 in Figure $3 a$ versus $c$. In contrast, performance under control conditions improved by an average of $0.16 \pm 0.14$ errors overnight. Similarly, on the novel maze, subjects given DMI committed $0.5 \pm 0.20$ more errors on the first three laps than on the last three laps the previous day. Controls, however, made $0.32 \pm 0.20$ fewer errors in the first three laps than the last laps of the previous day (MANOVA drug effect, $p=0.0217$ ). This overnight loss of memory under DMI was consistent across days of the experiment; there was no significant day effect $(p=$ $0.0825)$ or day by drug interaction ( $p=$ 0.1809), meaning that DMI affected memory retention on all days.

The DMI treatment condition also increased the utilization of nonhippocampal strategies to locate the positions of new food boxes, as revealed by a significant increase in errors after a $180^{\circ}$ maze rotation between laps 10 and 11 on both novel (Fig. $3 e ; p=0.0253$ ) and familiar (Fig. 3f; $p=0.0013$ ) mazes. The rotation pitted nonhippocampusdependent local cue use against hippocampus-dependent allocentric spatial map strategies. Although, control animals also made more errors on lap 11 than lap 10 on the familiar maze $(p=0.042)$, performance always remained within criterion ( $<1$ error/lap).

Spatial hippocampus-dependent consolidation of new learning and reconsolidation of the familiar maze were thus both attenuated by DMI treatment in a sleep-dependent manner, with the most relevant sleep metric being the length of time spent in the transition to REM sleep state during DMI after training each day. The deficit was due to a loss of consolidation of the memory rather than any working or short term memory impairments, and the deficit in spatial memory was compensated by a switch to procedural solutions as revealed in the increased errors after egocentric cues were rotated against the allocentric map.

\section{DMI spatial memory related to REM and TR suppression, not SWS}

Decreases in spatial maze performance under DMI treatment reflect memory consolidation or reconsolidation errors, and these errors were associated with REM and TR sleep changes. TR and REM sleep can be independently regulated (Fig. $2 f$ ); TR often occurs without a subsequent REM period, and REM sleep can occur with an abbreviated TR state.

Neither familiar nor novel maze spatial memory was related to the amounts of slow-wave sleep. The Pearson correlation $(R)$ of familiar and novel maze improvement versus SWS ranged from $-0.46(p=0.24)$ to $0.01(p=0.98)$ for correlations with SWS at baseline, SWS during DMI, and percent change in SWS between baseline and testing conditions.

\section{DMI procedural memory correlated to SWS}

In sharp contrast with the effects of DMI on hippocampusdependent learning performance, DMI treatment significantly improved performance on the procedural T-maze task over con-
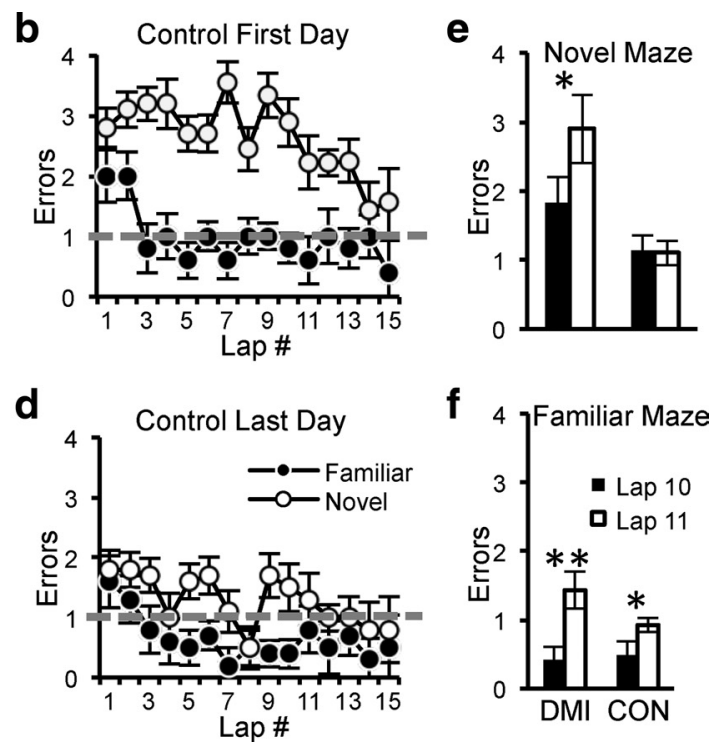

Figure 3. Within-session performance. $\boldsymbol{a}$ - $\boldsymbol{d}$, Lap-by-lap performance on the first $(\boldsymbol{a}, \boldsymbol{b})$ and last $(\boldsymbol{c}, \boldsymbol{d})$ days of eight-box 作 ( the novel $(\boldsymbol{e})$ and familiar $(\boldsymbol{f})$ mazes. CON, Control. Error rate for lap 10 (pre-maze rotation: black) versus lap e). ${ }^{*} p<0.05 ;{ }^{* *} p<0.01$.

trol treatment in both percent correct (repeated measures MANOVA $F=14.9, p=0.001$; Fig. $5 a$ ), and latency measures $(F=22.6, p=0.000$; Fig. $5 b)$ and correlated with the amounts of SWS during testing (Fig. $4 a$ ). There was no overall day effect $(F=$ $1.96, p=0.13)$, but a within-subjects day by group interaction was $(F=4.2, p=0.01)$ manifest in a nonlinear change in latency over days (quadratic $F=13, p=0.002$ ). Treated subjects with the largest percent increases in SWS during DMI administration performed well on the T-maze, whereas those with declines made far fewer correct choices (Fig. 4c). Although REM sleep during testing was not related to procedural task performance during DMI treatment (Fig. 4e,g), the percent REM sleep obtained during the prior week's baseline recordings was related to training week T-maze performance (Fig. $4 f$ ). The amount of REM sleep during baseline was inversely correlated with the percent change in SWS during DMI treatment $(r=0.8942, p=0.0066)$, as though DMI were replacing baseline REM sleep with SWS and those with higher baseline REM sleep left more sleep time after REM suppression for SWS to fill. The more REM sleep a subject showed during baseline, the higher the percent of SWS during DMI (Fig. $4 h$ ) and the better the procedural performance. Little relationship has been found between the amounts of REM sleep and nonREM sleep under normal conditions (Benington and Heller, 1994; Franken, 2002), so a relationship separated by days and experimental treatments and connected through learning is surprising.

The number of correct choices and the latency to trial completion were highly correlated, as expected $(r=-0.9533, p=$ 0.00025; Fig. 5c). Thus, the two sleep parameters, percent SWS at testing and percent REM sleep at baseline, that were correlated with the number of correct T-maze choices were also significantly and inversely correlated with latency to trial completion (Table 1). T-maze performance was not correlated with the amount of waking or TR sleep obtained during baseline or testing periods, although there was a trend for those rats that preserved or en- 

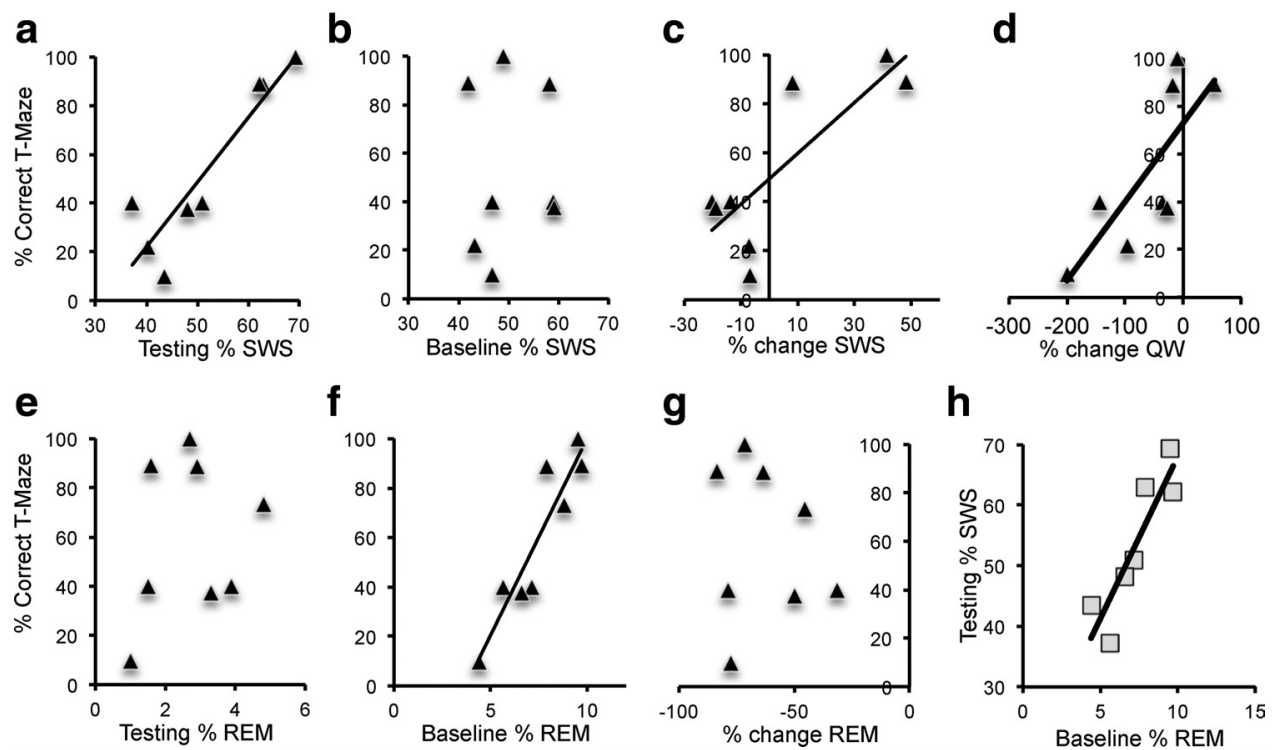

Figure 4. Performance on the T-maze procedural task. Relationship between T-maze performance and markers of SWS, REM sleep, and quiet waking (QW). Vertical axis represents percent correct T-maze choices, while the horizontal axis shows sleep state quantities over the first $12 \mathrm{~h}$ of recording time during the DMI test week, during circadian time-matched baseline sleep recording days, or changes from baseline to DMI-treated sleep recordings. All significant Pearson's correlations are noted by the presence of the fitted line. $\boldsymbol{a}$, Percent SWS versus T-maze performance during DMI test days. $\boldsymbol{b}$, Percent SWS during baseline versus T-maze performance on DMI test days. $\boldsymbol{c}$, T-maze performance versus percent change in SWS between baseline and DMI test days. $\boldsymbol{d}$, T-maze performance versus percent change in QW from baseline to DMI treatment days. $\boldsymbol{e}$, T-maze performance versus amount of REM sleep during the DMI test days. $\boldsymbol{f}$, T-maze performance on DMI test days versus percent REM sleep obtained during baseline training days. $\boldsymbol{g}$, Percent change in REM sleep from baseline to testing vs. T-maze performance. $\boldsymbol{h}$, Correlation between amount of baseline REM sleep and amount of SWS during testing.
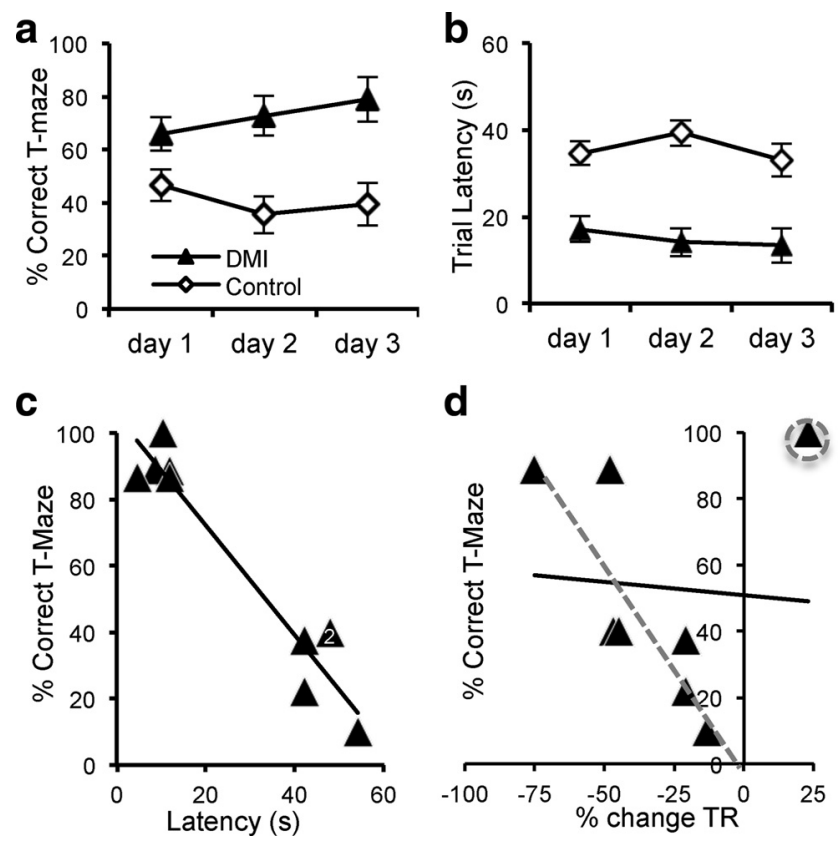

Figure 5. Learning curve for the T-maze. $\boldsymbol{a}$, T-maze performance over the first 3 days of testing under DMI treatment or control treatment conditions. Note DMI-treated animals showed more correct responses over all 3 days of testing. $\boldsymbol{b}$, Trial latency by treatment group. $\boldsymbol{c}$, Relationship between trial latency (horizontal axis) and performance (vertical axis) in DMI-treated animals ( $r=-0.9533$, $p=0.00025)$. Thenumber 2 in onetriangle denotes two rats with overlapping values. $\boldsymbol{d}$, Relationship between TR sleep suppression and T-maze performance. Black line represents non-significant correlation (all 8 animals, $p=$ n.s.). Dashed trend line represents correlation following removal of a single circled outlier $(n=7, r=-0.856, p=0.014)$.

hanced their amount of quiet wakefulness (which in the hippocampus looks like SWS) under DMI to also perform best on the procedural task $(r=0.772, p=0.025$; Fig. $4 d)$. DMI, then, increased procedural T-maze performance in a sleep-dependent fashion according to the amount of REM sleep during baseline and SWS during treatment.

\section{Discussion}

Overall, our hypothesis that DMI-suppressed REM sleep inhibits hippocampus-dependent learning and promotes compensatory nonhippocampal strategies that partially rescue within-session performance was supported. Surprisingly, we found that impairments in reversal learning were best correlated with declines in the TR state rather than decreases in REM sleep (Fig. 2e). Conversely, reconsolidation of a familiar memory was poorest in those with the highest retention of TR sleep during DMI testing (Fig. 2c), as though the processes during TR that best predict successful incorporation of novel information were unsupportive of the maintenance of an older schema. As predicted, reconsolidation was associated with the degree of REM sleep disturbance during antidepressant treatment (Fig. 2b). Procedural learning, however, was enhanced by DMI (Fig. $5 a, b)$, correlated with augmented SWS during testing (Fig. 4a,c), and, surprisingly, with the amount of baseline REM sleep (Fig. $4 f$ ).

These spatial and procedural learning results offer insight into how various sleep states may suppress or augment the consolidation of memories during sleep. Together, our findings and other studies discussed below suggest that the consolidation of novel learning, reconsolidation of familiar memories, and consolidation of procedural memories may involve routines differentially called during different sleep states, with novel memory consolidation occurring during TR (spindles), reconsolidation during REM sleep, and striatum-dependent procedural consolidation during SWS.

This is the first measure of reconsolidation memory under REM sleep deprivation of any kind, and the first test of antidepressant medication effects on the recall of familiar memories. Our results indicate that the sleep period following reactivation of a familiar schema is important for the reconsolidation process. 
The subjects used in the present study were normal rats rather than depressed humans. However, the profound effect of this standard desipramine dose on sleep and memory suggests that further careful testing should be conducted on sleep-dependent learning strategy selections in normal humans as well as those suffering with depression.

\section{REM sleep supports reconsolidation}

REM sleep deprivation is coincident with a decrease in hippocampal function in humans (Yoo et al., 2007). Induced neural activity during REM sleep has been shown to drive changes in synaptic weights (Bramham and Srebro, 1989). Reactivation of neurons encoding a memory network occurs during REM sleep (Pavlides and Winson, 1989) that supports both LTP during new learning and the reversal of LTP (depotentiation) for consolidated memories within the novelty encoding pathway (Poe et al., 2000). Thus, memories are likely revisited and reshaped during REM sleep outside of the original learning experience (Poe et al., 2010). The prevention of REM sleep by DMI also prevents all REM sleep-related processes, including hippocampal reactivation, REM theta, and theta phase activity that supports depotentiation. These results for learning agree with the effects seen with REM sleep deprivation on hippocampal function in vitro through loss of induction or maintenance of LTP (Davis et al., 2003), including reductions in excitability (McDermott et al., 2003). Without timely opportunity to enter REM sleep and accomplish synapse-specific strengthening or weakening, consolidation and reconsolidation could be impaired. In the closest study to ours, a once daily $10 \mathrm{mg} / \mathrm{kg}$ dose testing rats on an eight-arm radial maze found similar impairments in learning (Burgos et al., 2005); presumably REM sleep was also suppressed in their study.

\section{TR sleep supports novel learning and impairs reconsolidation}

Our data indicate that consolidation of novel learning and reconsolidation of familiar knowledge are both proportional to the amount of TR sleep, though in opposite directions. Reactivation of hippocampal neurons and interaction with prefrontal cortex is uniquely intense during sleep spindles, possibly supporting memory consolidation (Siapas and Wilson, 1998; Siapas et al., 2005). Sleep spindles, which characterize the TR state, do not occur when NA, acetylcholine (ACh), or serotonin is present in the thalamus (Lee and McCormick, 1996). The DMI block of NA reuptake during TR could decimate spindles and the hippocampal-forebrain reverberations that accompany them. Increases or a relative preservation of TR may maintain sufficient spindlerelated reverberation to incorporate novel information into established, consolidated synaptic memory networks. Maintenance of familiar memories in the face of daily novel learning suffered in subjects with higher percent TR during DMI. Finally, like REM sleep, TR is a state where $\mathrm{P}$-waves are present, and $\mathrm{P}$-wave density during TR and REM sleep have been strongly correlated with learning novel relationships (Datta, 2000; Datta et al., 2004). Taken together, perhaps the strong correlation between novel learning and TR and the negative association with familiar memories is linked through increased novel interference with familiar map memories. More studies would be needed to establish a causal link. These results also agree with recent findings on the function of sleep spindles (Fogel and Smith, 2011) in humans. However, little is known about this transient sleep state and its interaction with mechanisms of learning (Poe et al., 2010).

\section{SWS during consolidation supports dorsal striatal learning}

Procedural learning was, in our hands, enhanced proportionately with the degree of increase in SWS under DMI treatment. SWSdependent consolidation in the striatum may depend on processes such as firing rate/pattern changes (Bordi et al., 1993) or low ACh levels (Gadea-Ciria et al., 1973; Havekes et al., 2011) during SWS. Increases in SWS have been shown to correlate with other procedural learning tasks such as soccer playing and tumbling on a trampoline (Buchegger and Meier-Koll, 1988; Doyon et al., 2009; Debas et al., 2010; Debarnot et al., 2011). Higher slow-wave activity $(0-3 \mathrm{~Hz})$ during SWS has also been shown to correlate with procedural and implicit learning (Huber et al., 2004; Göder et al., 2006). Similarly, the quiet wakefulness correlation with improved procedural memory also supports this theory, since the hippocampus is also "offline" in the same large irregular activity, multiunit burst mode characteristic of SWS during quiet waking (Suzuki and Smith, 1985).

\section{Basal REM sleep supports dorsal striatal procedural learning}

We found that the amount of baseline REM sleep correlated to striatum-dependent fixed choice T-maze task performance. Markowska et al. also found a positive correlation between the amounts of baseline REM sleep and striatal learning performance measuring the swim path length on a visible platform Morris water maze (Markowska et al.,1989). Interestingly, amounts of serotonin in the striatal caudate correlate with the amounts of baseline REM sleep, and the numbers of 5HT-2 receptors in the caudate correlate with visible platform water maze task performance, suggesting some function for baseline REM sleep and caudate serotonin levels in motor learning (Markowska et al., 1989). It would be interesting to test whether basal amounts of REM sleep correlate with all striatum-dependent learning tasks and whether serotonin is key to that involvement.

\section{DMI sleep effects parallel human antidepressant use}

These findings support those found by Rasch et al. (2009), who demonstrated that antidepressants enhanced procedural learning. Their study and ours indicate that the effects of REM suppression on striatum-dependent procedural memory are quite different than those on hippocampus-dependent spatial memory. Some antidepressants, but not others, have been reported to improve memory function (Levkovitz et al., 2002). Our study suggests that the outcomes may depend on the sleep effects of the particular antidepressant and the memory type (e.g., striatal dependent or hippocampus dependent) tested.

Antidepressant administration is an effective method of reducing REM sleep (Mayers and Baldwin, 2005). We were able to use a dose resulting in transient REM reduction that fully dissipated by $10 \mathrm{~h}$ and then returned to normal. We produced this effect repeatedly across all 4 days of treatment. Both the pharmokinetics (despiramine's half-life of $4.6 \mathrm{~h}$; Kozisek et al., 2007) and the general effects on sleep $(8-10 \mathrm{~h})$ architecture we observed indicate that DMI's action had ceased many hours before the following day's run. Thus, DMI did not likely mediate the change in daily performance directly, but rather worked during the postlearning consolidation period when DMI actively suppressed REM sleep.

Chronic desipramine suppresses human REM sleep most strongly in the initial 3 weeks, after which REM sleep quantity begins to rebound slightly although never reaching pretreatment amounts (Shipley et al., 1985). If the REM sleep state itself is responsible for hippocampal memory consolidation, then consolidation-related performance deficits may improve somewhat several weeks into 
treatment, coincident with a partial restoration of REM sleep. This hypothesis remains to be tested. Additionally, we determined that although REM sleep suppressing antidepressant treatments impaired hippocampus-dependent learning, alternative strategies were augmented concomitantly, much as has been found in other animal studies with REM restriction (Bjorness et al., 2005; Hagewoud et al., 2010; Walsh et al., 2011). Such alternative learning strategies could limit REM deprivation-dependent learning deficits. Future studies could offer insight into whether other antidepressants also negatively affect hippocampus-dependent memories while benefiting procedural learning and whether patients taking REM-suppressing antidepressants may benefit from selecting REM sleep immune non-hippocampal strategies to solve learning problems at hand.

These results, taken together with other published studies, lead us to propose a novel set of sleep stage associations important for the consolidation of sometimes competing memory tasks, with REM sleep possibly being more conducive to reconsolidating familiar memories, TR sleep important for consolidating novel memories at the expense of familiar schema, and SWS enhancing procedural strategies.

\section{References}

Aston-Jones G, Bloom FE (1981) Activity of norepinephrine-containing locus coeruleus neurons in behaving rats anticipates fluctuations in the sleep-waking cycle. J Neurosci 1:876-886.

Baumann P, Gaillard JM, Perey M, Justafré JC, Le P (1983) Relationships between brain concentrations of desipramine and paradoxical sleep inhibition in the rat. J Neural Transm 56:105-116.

Benington JH, Heller HC (1994) REM-sleep timing is controlled homeostatically by accumulation of REM-sleep propensity in non-REM sleep. Am J Physiol 266:R1992-R2000.

Bjorness TE, Riley BT, Tysor MK, Poe GR (2005) REM restriction persistently alters strategy used to solve a spatial task. Learn Mem 12:352-359.

Bordi F, LeDoux J, Clugnet MC, Pavlides C (1993) Single-unit activity in the lateral nucleus of the amygdala and overlying areas of the striatum in freely behaving rats: rates, discharge patterns, and responses to acoustic stimuli. Behav Neurosci 107:757-769.

Bramham CR, Srebro B (1989) Synaptic plasticity in the hippocampus is modulated by behavioral state. Brain Res 493:74-86.

Brooks JO, Hoblyn JC (2007) Neurocognitive costs and benefits of psychotropic medications in older adults. J Geriatr Psychiatry Neurol 20:199-214.

Buchegger J, Meier-Koll A (1988) Motor learning and ultradian sleep cycle: an electroencephalographic study of trampoliners. Percept Mot Skills 67:635-645.

Burgos H, Mardones L, Campos M, Castillo A, Fernández V, Hernández A (2005) Chronic treatment with clomipramine and desipramine induces deficit in long-term visuo-spatial memory of rats. Int J Neurosci 115:47-54.

Chang Q, Gold PE (2003) Intra-hippocampal lidocaine injections impair acquisition of a place task and facilitate acquisition of a response task in rats. Behav Brain Res 144:19-24.

Datta S (2000) Avoidance task training potentiates phasic pontine-wave density in the rat: a mechanism for sleep-dependent plasticity. J Neurosci 20:8607-8613.

Datta S, Mavanji V, Ulloor J, Patterson EH (2004) Activation of phasic pontine-wave generator prevents rapid eye movement sleep deprivationinduced learning impairment in the rat: a mechanism for sleepdependent plasticity. J Neurosci 24:1416-1427.

Davis CJ, Harding JW, Wright JW (2003) REM sleep deprivation-induced deficits in the latency-to-peak induction and maintenance of long-term potentiation within the CA1 region of the hippocampus. Brain Res 973:293-297.

Debarnot U, Castellani E, Valenza G, Sebastiani L, Guillot A (2011) Daytime naps improve motor imagery learning. Cogn Affect Behav Neurosci 11:541-550.

Debas K, Carrier J, Orban P, Barakat M, Lungu O, Vandewalle G, Hadj Tahar A, Bellec P, Karni A, Ungerleider LG, Benali H, Doyon J (2010) Brain plasticity related to the consolidation of motor sequence learning and motor adaptation. Proc Natl Acad Sci U S A 107:17839-17844.
Doyon J, Korman M, Morin A, Dostie V, Hadj Tahar A, Benali H, Karni A, Ungerleider LG, Carrier J (2009) Contribution of night and day sleep vs. simple passage of time to the consolidation of motor sequence and visuomotor adaptation learning. Exp Brain Res 195:15-26.

Fogel SM, Smith CT (2011) The function of the sleep spindle: a physiological index of intelligence and a mechanism for sleep-dependent memory consolidation. Neurosci Biobehav Rev 35:1154-1165.

Franken P (2002) Long-term vs. short-term processes regulating REM sleep. J Sleep Res 11:17-28.

Gadea-Ciria M, Stadler H, Lloyd KG, Bartholini G (1973) Acetylcholine release within the cat striatum during the sleep-wakefulness cycle. Nature 243:518-519.

Göder R, Aldenhoff JB, Boigs M, Braun S, Koch J, Fritzer G (2006) Delta power in sleep in relation to neuropsychological performance in healthy subjects and schizophrenia patients. J Neuropsychiatry Clin Neurosci 18:529-535.

Goodman LS, Gilman A, Brunton LL, Lazo JS, Parker KL (2006) Goodman and Gilman's the pharmacological basis of therapeutics, Ed 11. New York: McGraw-Hill.

Gross BA, Walsh CM, Turakhia AA, Booth V, Mashour GA, Poe GR (2009) Open-source logic-based automated sleep scoring software using electrophysiological recordings in rats. J Neurosci Methods 184:10-18.

Hagewoud R, Havekes R, Tiba PA, Novati A, Hogenelst K, Weinreder P, Van der Zee EA, Meerlo P (2010) Coping with sleep deprivation: shifts in regional brain activity and learning strategy. Sleep 33:1465-1473.

Havekes R, Abel T, Van der Zee EA (2011) The cholinergic system and neostriatal memory functions. Behav Brain Res 221:412-423.

Huber R, Ghilardi MF, Massimini M, Tononi G (2004) Local sleep and learning. Nature 430:78-81.

Jones BE, Yang TZ (1985) The efferent projections from the reticular formation and the locus coeruleus studied by anterograde and retrograde axonal transport in the rat. J Comp Neurol 242:56-92.

Katsuki H, Izumi Y, Zorumski CF (1997) Noradrenergic regulation of synaptic plasticity in the hippocampal CA1 region. J Neurophysiol 77:3013-3020.

Kemp A, Manahan-Vaughan D (2004) Hippocampal long-term depression and long-term potentiation encode different aspects of novelty acquisition. Proc Natl Acad Sci U S A 101:8192-8197.

Kozisek ME, Deupree JD, Burke WJ, Bylund DB (2007) Appropriate dosing regimens for treating juvenile rats with desipramine for neuropharmacological and behavioral studies. J Neurosci Methods 163:83-91.

Lee KH, McCormick DA (1996) Abolition of spindle oscillations by serotonin and norepinephrine in the ferret lateral geniculate and perigeniculate nuclei in vitro. Neuron 17:309-321.

Levkovitz Y, Caftori R, Avital A, Richter-Levin G (2002) The SSRIs drug Fluoxetine, but not the noradrenergic tricyclic drug Desipramine, improves memory performance during acute major depression. Brain Res Bull 58:345-350.

Manahan-Vaughan D, Braunewell KH (1999) Novelty acquisition is associated with induction of hippocampal long-term depression. Proc Natl Acad Sci U S A 96:8739-8744.

Markowska AL, Stone WS, Ingram DK, Reynolds J, Gold PE, Conti LH, Pontecorvo MJ, Wenk GL, Olton DS (1989) Individual differences in aging: behavioral and neurobiological correlates. Neurobiol Aging 10: 31-43.

Mayers AG, Baldwin DS (2005) Antidepressants and their effect on sleep. Hum Psychopharmacol 20:533-559.

McDermott CM, LaHoste GJ, Chen C, Musto A, Bazan NG, Magee JC (2003) Sleep deprivation causes behavioral, synaptic, and membrane excitability alterations in hippocampal neurons. J Neurosci 23:9687-9695.

McGaugh JL (2000) Memory-a century of consolidation. Science 287: $248-251$

Morris RG, Hagan JJ, Rawlins JN (1986) Allocentric spatial learning by hippocampectomised rats: a further test of the "spatial mapping" and "working memory" theories of hippocampal function. Q J Exp Psychol B 38:365-395

Nader K, Hardt O (2009) A single standard for memory: the case for reconsolidation. Nat Rev Neurosci 10:224-234.

Nicola SM, Malenka RC (1998) Modulation of synaptic transmission by dopamine and norepinephrine in ventral but not dorsal striatum. J Neurophysiol 79:1768-1776.

Packard MG, McGaugh JL (1996) Inactivation of hippocampus or caudate 
nucleus with lidocaine differentially affects expression of place and response learning. Neurobiol Learn Mem 65:65-72.

Pavlides C, Winson J (1989) Influences of hippocampal place cell firing in the awake state on the activity of these cells during subsequent sleep episodes. J Neurosci 9:2907-2918.

Poe GR, Nitz DA, McNaughton BL, Barnes CA (2000) Experiencedependent phase-reversal of hippocampal neuron firing during REM sleep. Brain Res 855:176-180.

Poe GR, Thompson CM, Riley BT, Tysor MK, Bjorness TE, Steinhoff BP, Ferluga ED (2002) A spatial memory task appropriate for electrophysiological recordings. J Neurosci Methods 121:65-74.

Poe GR, Walsh CM, Bjorness TE (2010) Cognitive neuroscience of sleep. Prog Brain Res 185:1-19.

Rasch B, Pommer J, Diekelmann S, Born J (2009) Pharmacological REM sleep suppression paradoxically improves rather than impairs skill memory. Nat Neurosci 12:396-397.

Restle F (1957) Discrimination of cues in mazes: a resolution of the placevs.-response question. Psychol Rev 64:217-228.

Roberson T, Riley B, Poe G (2004) REM deprivation significantly reduces a hippocampally lesioned rat's ability to acquire a spatial learning task. J Sleep Res [Suppl 13]:611.

Shipley JE, Kupfer DJ, Griffin SJ, Dealy RS, Coble PA, McEachran AB, Grochocinski VJ, Ulrich R, Perel JM (1985) Comparison of effects of desipramine and amitriptyline on EEG sleep of depressed patients. Psychopharmacology 85:14-22.

Siapas AG, Wilson MA (1998) Coordinated interactions between hippocampal ripples and cortical spindles during slow-wave sleep. Neuron 21:1123-1128.
Siapas AG, Lubenov EV, Wilson MA (2005) Prefrontal phase locking to hippocampal theta oscillations. Neuron 46:141-151.

Smith CT, Conway JM, Rose GM (1998) Brief paradoxical sleep deprivation impairs reference, but not working, memory in the radial arm maze task. Neurobiol Learn Mem 69:211-217.

Suzuki SS, Smith GK (1985) Single-cell activity and synchronous bursting in the rat hippocampus during waking behavior and sleep. Exp Neurol 89:71-89.

Thomas MJ, Moody TD, Makhinson M, O’Dell TJ (1996) Activitydependent beta-adrenergic modulation of low frequency stimulation induced LTP in the hippocampal CA1 region. Neuron 17:475-482.

Tolman EC, Ritchie BF, Kalish D (1946) Studies in spatial learning; place learning versus response learning. J Exp Psychol 36:221-229.

van der Meulen JA, Bilbija L, Joosten RN, de Bruin JP, Feenstra MG (2003) The NMDA-receptor antagonist MK-801 selectively disrupts reversal learning in rats. Neuroreport 14:2225-2228.

Walsh CM, Booth V, Poe GR (2011) Spatial and reversal learning in the Morris water maze are largely resistant to six h of REM sleep deprivation following training. Learn Mem 18:422-434.

Wimmer F, Hoffmann RF, Bonato RA, Moffitt AR (1992) The effects of sleep deprivation on divergent thinking and attention processes. J Sleep Res 1:223-230.

Xu L, Anwyl R, Rowan MJ (1998) Spatial exploration induces a persistent reversal of long-term potentiation in rat hippocampus. Nature 394: 891-894.

Yoo SS, Hu PT, Gujar N, Jolesz FA, Walker MP (2007) A deficit in the ability to form new human memories without sleep. Nat Neurosci 10:385-392. 